\title{
Procedure volume and mortality after surgical lung biopsy in interstitial lung disease
}

\author{
Jolene H. Fisher ${ }^{1}$, Shane Shapera ${ }^{1}$, Teresa To ${ }^{2,3,4}$, Theodore K. Marras ${ }^{1}$, \\ Andrea Gershon ${ }^{1,2,3,4}$ and Sharon Dell ${ }^{3,4}$ \\ Affiliations: \\ ${ }^{1}$ Dept of Medicine, University of Toronto, Toronto, ON, Canada. \\ ${ }^{2}$ Institute for Clinical Evaluative Sciences, Toronto, ON, Canada. \\ ${ }^{3}$ Child Health Evaluative Sciences, The Hospital for Sick Children, Toronto, ON, Canada. \\ ${ }^{4}$ Dalla Lana School of Public Health, University of Toronto, Toronto, ON, Canada.
}

Correspondence:

Jolene H. Fisher, University Health Network, 9N-935, 585 University Avenue, University of Toronto, Toronto, ON M5G 2N2, Canada.

E-mail: jolene.fisher@uhn.ca

@ERSpublications

Higher hospital surgical lung biopsy volume was associated with lower post-operative mortality in patients with ILD. Mortality for nonelective procedures was much higher than elective procedures. http://ow.ly/GrXV30mNJuJ

Cite this article as: Fisher JH, Shapera S, To T, et al. Procedure volume and mortality after surgical lung biopsy in interstitial lung disease. Eur Respir J 2019; 53: 1801164 [https://doi.org/10.1183/ 13993003.01164-2018].

ABSTRACT Surgical volume-outcome relationships are well established but have not been studied in patients with interstitial lung disease (ILD) undergoing surgical lung biopsy (SLB). Our study objective was to determine if hospital SLB volume is associated with post-operative mortality in patients with ILD.

A cohort study using administrative, population-based data from Ontario, Canada was performed in adults with ILD who underwent a SLB between 2001 and 2014. The association between yearly hospital SLB volume and 30-day post-operative mortality was assessed using multilevel logistic regression modelling.

3057 surgical lung biopsies for ILD were performed during the study period with a median (interquartile range) yearly hospital volume of 73 (34-143) procedures. 30-day mortality was $7.1 \%, 20.2 \%$ and $1.9 \%$ in overall, nonelective and elective patients, respectively. Higher yearly hospital SLB volume was associated with lower odds of 30-day post-operative mortality after adjusting for patient characteristics (OR 0.84, 95\% CI 0.73-0.97; $\mathrm{p}=0.02$ ), with the association appearing stronger for nonelective versus elective procedures (OR 0.84, 95\% CI $0.69-1.02$; $\mathrm{p}=0.08$ versus OR $0.94,95 \%$ CI $0.74-1.18$; $\mathrm{p}=0.57$ ).

Higher yearly hospital SLB volume was associated with lower post-operative mortality in patients with ILD, with the association appearing to be mainly driven by nonelective cases. SLB mortality was significantly higher for nonelective cases. 


\section{Introduction}

Interstitial lung diseases (ILDs) are a diverse collection of lung diseases with varying epidemiology, clinical course and management [1-3]. Determining the correct ILD subtype is essential for appropriate clinical decision making, patient counselling and meaningful research. The diagnosis of a specific ILD is made based on a combination of clinical, radiological and sometimes pathological features [1-4]. Transbronchial biopsy is usually inadequate for definitive diagnosis of ILDs and the role of cryobiopsy remains controversial, often necessitating a surgical lung biopsy (SLB) when histopathology is required [1-4]. Although SLB is the preferred method of obtaining pathological samples in patients with ILD, prior research suggests substantial and extremely variable post-operative mortality rates, ranging from $3 \%$ to $16.7 \%$ at $30-60$ days [5-8]. The reasons behind such wide variability in SLB mortality in the literature are not well studied to date. Individual studies have found certain patient clinical characteristics to be associated with higher mortality $[5-7,9,10]$; however, it is unclear if these can completely account for the mortality variability seen in the literature.

Surgical volume-outcome relationships are well established and have been studied for many procedures. A US study of 2.5 million patients undergoing one of 14 procedure types (six cardiovascular and eight cancer related) found that mortality consistently decreased as procedure volume increased [11]. A Canadian Institute for Health Information-commissioned systematic review of 161 volume-outcome relationship studies found that higher volume was associated with better outcome in the majority, although the strength of association varied with procedure type [12]. Several other studies and systematic reviews have shown similar findings, with higher volumes often being associated with better outcomes [13, 14]. The volume-outcome relationship has been used as evidence to support the regionalisation of care for certain conditions, such as lung cancer, in Canada $[11,15]$.

ILDs comprise a group of complex disorders, often cared for in expert centres, and hospitals with a higher volume of SLBs are likely to have more experience with these patients. This variation in experience could theoretically result in pre-, intra- and post-operative care differences between hospitals that influence mortality. Factors including surgeon and anaesthetist expertise, patient selection, availability of noninvasive techniques (equipment and staffing), and experience of the post-operative care team may all affect patient outcome. Therefore, the aim of this study was to evaluate the association between hospital SLB volume and post-operative mortality in patients with ILD undergoing SLB using large, population-based databases. Our pre-specified hypothesis was that higher hospital SLB volume would be associated with lower 30-day mortality after SLB in patients with ILD.

\section{Methods}

\section{Study design and setting}

An observational cohort study using administrative, population-based data available through the Institute for Clinical Evaluative Sciences (ICES) was performed in Ontario, Canada. Ontario is the single insurer for a universal healthcare system which provides essential healthcare services to Ontario's 13.6 million residents. As a result, healthcare data is collected and maintained on almost all of the province's residents. This study was approved by the institutional review board of the University Health Network (Toronto, ON, Canada).

\section{Study population}

All patients with a first admission for SLB in Ontario between April 2001 and March 2014 were identified using Canadian Classification of Health Interventions (CCI) and Canadian Classification of Diagnostic, Therapeutic and Surgical Procedures (CCP) codes (supplementary table S1) and screened for eligibility. The beginning of the study period was chosen based on the earliest date that all key study variables were collected in ICES databases. Patients were included if they 1) underwent a SLB, 2) were $\geqslant 18$ years of age and 3) had a diagnosis of ILD any time prior to or 1 year after SLB. International Classification of Diseases-9 or -10 codes were used to define ILD (supplementary table S2). Individuals with lung cancer and those with CCI or CCP codes for pneumonectomy, lobectomy and lung transplant were excluded (supplementary table S3).

\section{Data sources}

All data were ascertained by deterministic linkage of ICES databases using unique encrypted patient identifiers (see the supplementary material for database descriptions).

\section{Exposure, outcome and covariable definitions}

The primary exposure was defined as the number of SLBs performed at the hospital in the fiscal year of the index SLB. The primary outcome was 30-day mortality after SLB. Procedure type was defined as open 
thoracotomy versus video-assisted thoracoscopic surgery (VATS), and was determined using CCP codes prior to 2002 and CCI codes from 2002 onwards (supplementary table S1).

Covariates included in analyses were selected a priori based on clinical relevance and prior evidence, and included age, sex, long-term oxygen therapy, Charlson Comorbidity Index, socioeconomic status (as determined by income quintile), procedure type, procedure year and nonelective versus elective procedure. Long-term oxygen therapy was defined as continuous supplemental oxygen for home use. The Charlson Comorbidity Index is a validated tool using patient comorbidities to predict 1-year mortality in adults [16]. Nonelective procedure was defined as a SLB in a patient that was admitted to hospital prior to the procedure date. Patients who were admitted to hospital on the day of SLB were considered elective.

\section{Analysis}

Population characteristics by hospital SLB volume were described using mean with standard deviation, median (interquartile range (IQR)), frequencies and proportions, as appropriate.

To account for the hierarchical nature of healthcare data and clustering by hospital, multilevel logistic regression was used evaluate the association between hospital SLB volume and 30-day post-operative mortality. A series of two-level models was fitted with three models specified for the outcome (null, hospital-level covariate, and hospital- and patient-level covariates). The intraclass correlation coefficient (ICC) was calculated to determine the amount of variability observed in 30-day mortality attributable to between-hospital differences. The Hosmer-Lemeshow goodness-of-fit test and the c-statistic were used to evaluate model fit. The primary analysis was stratified by nonelective and elective patients, and hospital SLB volume was secondarily modelled using quartiles to identify a volume threshold (additional details available in the supplementary material). Statistical significance was defined by a two-tailed p-value $<0.05$. Analyses were performed using SAS version 9.4 (SAS Institute, Cary, NC, USA).

\section{Results}

3057 patients met study criteria and were included in the final cohort (figure 1). There were 216 deaths within 30 days after SLB, with an overall mortality of $7.1 \%$ for the entire study cohort. Mortality rate varied by procedure year, but in general remained similar over time (figure 2). 174 deaths occurred in nonelective procedures, with an overall mortality in this group of $20.2 \%$. Among elective procedures, there were 42 deaths with an overall mortality of $1.9 \%$. Median (IQR) time to death was 12 (6-18) days. Median (IQR) hospital length of stay was 3 (2-7) days. Readmission within 30 days of procedure occurred in 331 patients $(10.8 \%)$.

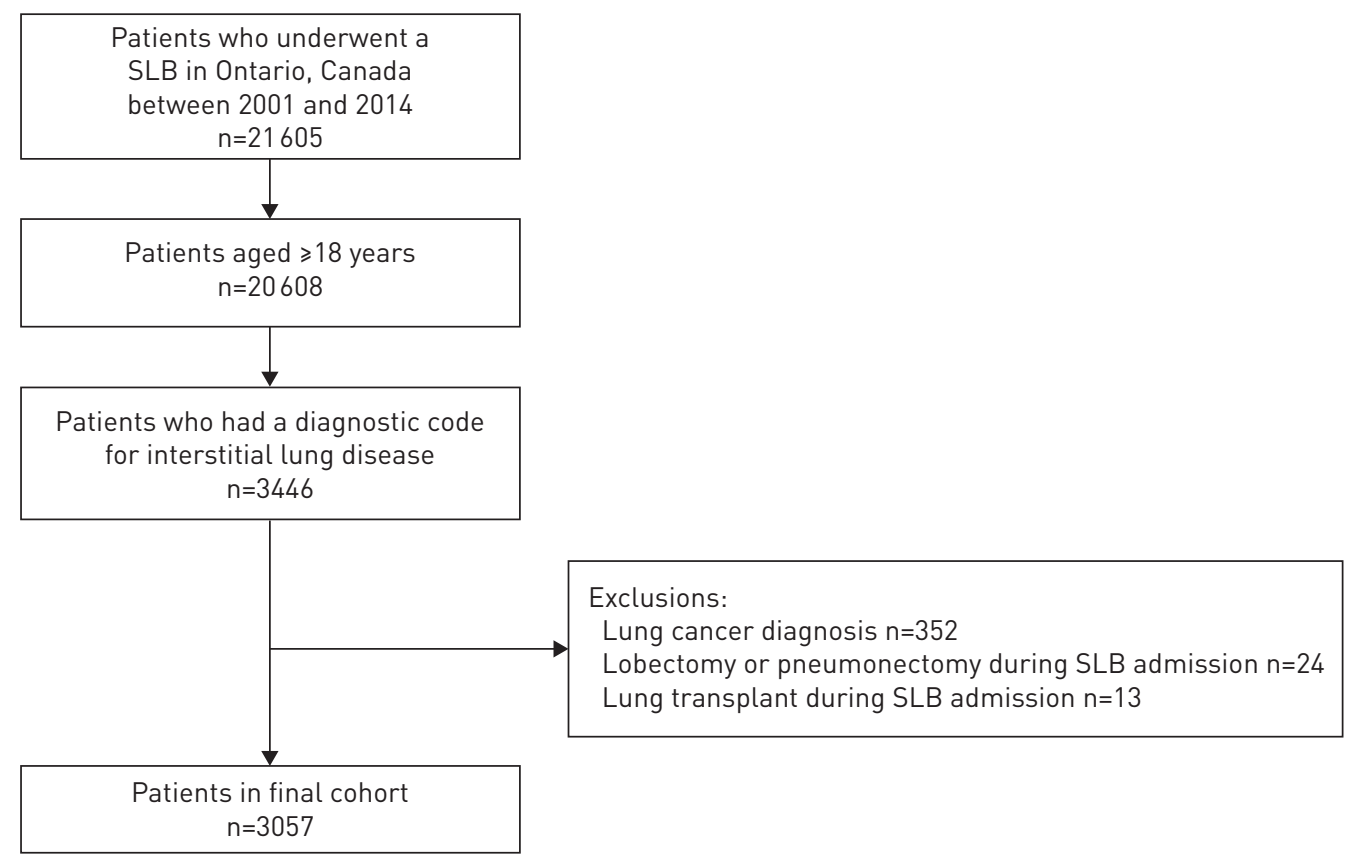

FIGURE 1 Study cohort selection. SLB: surgical lung biopsy. Of the 17162 patients who underwent SLB and did not have a diagnostic code for interstitial lung disease, 8345 had a diagnosis of lung cancer in the Ontario Cancer Registry. 
Patient characteristics

Patient characteristics overall, stratified by SLB volume quartile, and stratified by nonelective and elective procedure are summarised in table 1 and supplementary table S4. Most patients were older adults, with similar proportions of males and females, and a low Charlson Comorbidity Index. The fourth (highest) SLB volume quartile had the highest proportion of elective procedures. Age, sex and Charlson Comorbidity Index were similar throughout the study period; however, long-term oxygen therapy appeared to increase over time. Individual comorbidity data is shown in supplementary table S5.

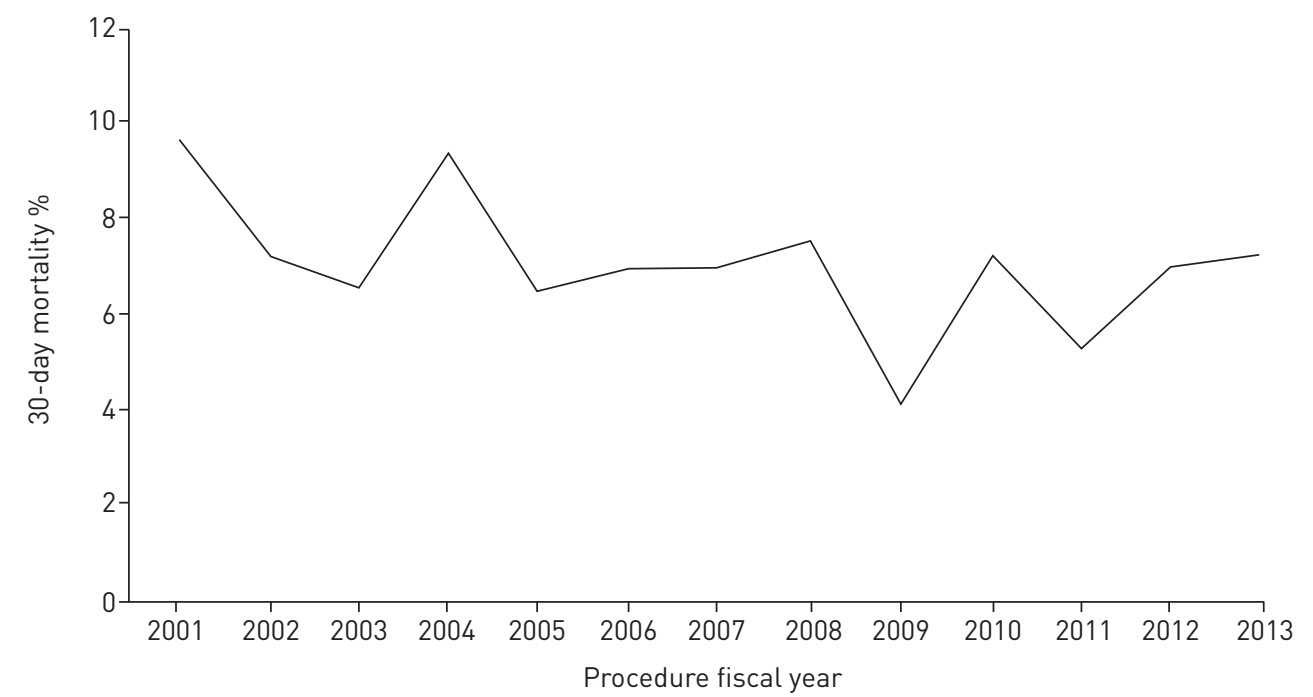

FIGURE 2 30-day mortality after surgical lung biopsy in interstitial lung disease patients by procedure fiscal year.

TABLE 1 Baseline characteristics of patients with interstitial lung disease undergoing surgical lung biopsy (SLB) by hospital surgical volume

\begin{tabular}{|c|c|c|c|c|c|}
\hline & Overall & $\begin{array}{c}\text { Quartile } 1 \\
\text { (1-34 SLBs } \\
\text { per year] }\end{array}$ & $\begin{array}{c}\text { Quartile } 2 \\
\text { (35-73 SLBs } \\
\text { per year) }\end{array}$ & $\begin{array}{c}\text { Quartile } 3 \\
\text { (74-143 SLBs } \\
\text { per year) }\end{array}$ & $\begin{array}{c}\text { Quartile } 4 \\
\text { [144-258 SLBs } \\
\text { per year] }\end{array}$ \\
\hline 30-day mortality & $216(7.1)$ & 78 (10.0) & $52(6.8)$ & $64(7.8)$ & $22(3.2)$ \\
\hline \multicolumn{6}{|l|}{ Age group years } \\
\hline $18-29$ & $96(3.1)$ & $23(2.9)$ & $18(2.4)$ & $33(4.0)$ & $22(3.2)$ \\
\hline $50-59$ & $827(27.1)$ & $222(28.4)$ & $189(24.8)$ & $212(25.9)$ & $204(29.4)$ \\
\hline $60-69$ & $861(28.2)$ & $219(28.0)$ & $222(29.1)$ & $234(28.6)$ & $186(26.8)$ \\
\hline$\geqslant 70$ & $650(21.3)$ & $185(23.7)$ & $147(19.3)$ & $170(20.8)$ & $148(21.3)$ \\
\hline Male sex & $1582(51.8)$ & 413 (52.8) & 391 (51.3) & 436 (53.3) & 342 (49.3) \\
\hline \multicolumn{6}{|l|}{ Income quintile } \\
\hline 1 (lowest) & $602(19.8)$ & $152(19.5)$ & $178(23.4)$ & $153(18.8)$ & 119 (17.2) \\
\hline 0 & $1682(55.1)$ & 416 (53.3) & $432(56.7)$ & $421(51.5)$ & 413 (59.8) \\
\hline 1 & $702(23.0)$ & $180(23.1)$ & $165(21.7)$ & $205(25.1)$ & $152(22.0)$ \\
\hline 2 & $307(10.1)$ & 89 (11.4) & 83 (10.9) & $88(10.8)$ & $47(6.8)$ \\
\hline$\geqslant 3$ & $360(11.8)$ & 96 (12.3) & $82(10.8)$ & $103(12.6)$ & $79(11.4)$ \\
\hline Charlson Comorbidity Index overall & $0(0-1)$ & $0(0-1)$ & $0(0-1)$ & $0(0-1)$ & $0(0-1)$ \\
\hline Long-term oxygen therapy & $210(6.9)$ & $48(6.1)$ & $43(5.6)$ & $65(8.0)$ & $54(7.8)$ \\
\hline Nonelective & $860(28.1)$ & $284(36.3)$ & $223(29.2)$ & $238(29.1)$ & $115(16.6)$ \\
\hline
\end{tabular}

Data are presented $\mathrm{n}, \mathrm{n}(\%)$ or median (interquartile range). 
TABLE 2 Surgical lung biopsy (SLB) details for patients with interstitial lung disease by hospital surgical volume

\begin{tabular}{lccccc} 
& Overall & $\begin{array}{c}\text { Quartile 1 } \\
\text { (1-34 SLBs } \\
\text { per year) }\end{array}$ & $\begin{array}{c}\text { Quartile 2 } \\
\text { (35-73 SLBs } \\
\text { per year) }\end{array}$ & $\begin{array}{c}\text { Quartile 3 } \\
\text { (74-143 SLBs } \\
\text { per year) }\end{array}$ & $\begin{array}{c}\text { Quartile 4 } \\
\text { (144-258 SLBs } \\
\text { per year) }\end{array}$ \\
\hline Subjects & 3057 & 782 & 763 & 818 & 694 \\
Open thoracotomy & $1158(37.9)$ & $321(41.1)$ & $273(35.8)$ & $312(38.1)$ & $252(36.3)$ \\
Yearly hospital SLB volume & $73(34-143)$ & $23(13-30)$ & $50(41-64)$ & $115(96-137)$ & $208(170-220)$ \\
Total hospital SLB volume & $1219(462-2252)$ & $306(219-514)$ & $655(516-1219)$ & $1575(1453-2252)$ & $2345(2082-3681)$
\end{tabular}

Data are shown as $\mathrm{n}, \mathrm{n}(\%)$ or median (interquartile range).

Procedural details for the overall cohort and stratified by nonelective and elective procedure are summarised in table 2 and supplementary table S6. VATS was more common than open thoracotomy during the study period in the overall cohort, but was less common in nonelective procedures. Median (IQR) yearly and cumulative hospital SLB volume was 73 (34-143) and 1219 (462-2252), respectively.

\section{Primary outcome}

Results of the full multilevel model for 30-day post-operative mortality in the overall cohort and stratified by nonelective and elective patients are shown in table 3. Higher yearly hospital SLB volume was significantly associated with lower odds of 30-day post-operative mortality in the overall cohort (OR 0.84 , 95\% CI $0.73-0.97$; $\mathrm{p}=0.02$, unit for OR is per $50 \mathrm{SLBs}$ ), after adjusting for patient-level characteristics. In other words, for every additional 50 SLBs performed per year, the odds of dying within 30 days of the procedure decreased by $16 \%$. After stratification by nonelective and elective patients, the odds ratio for yearly hospital SLB volume was unchanged in nonelective patients compared with the overall cohort, although the $95 \%$ confidence intervals widened slightly and the p-value was no longer significant (OR 0.84, 95\% CI 0.69-1.02; $\mathrm{p}=0.08$, unit for OR is per $50 \mathrm{SLBs}$ ). Among elective patients, hospital SLB volume was no longer significantly associated with 30 -day post-operative mortality and the odds ratio increased

TABLE 3 Full multilevel models ${ }^{\#}$ of the association between yearly hospital surgical lung biopsy (SLB) volume and 30-day post-operative mortality in patients with interstitial lung disease undergoing SLB in the overall cohort and stratified by nonelective and elective patients

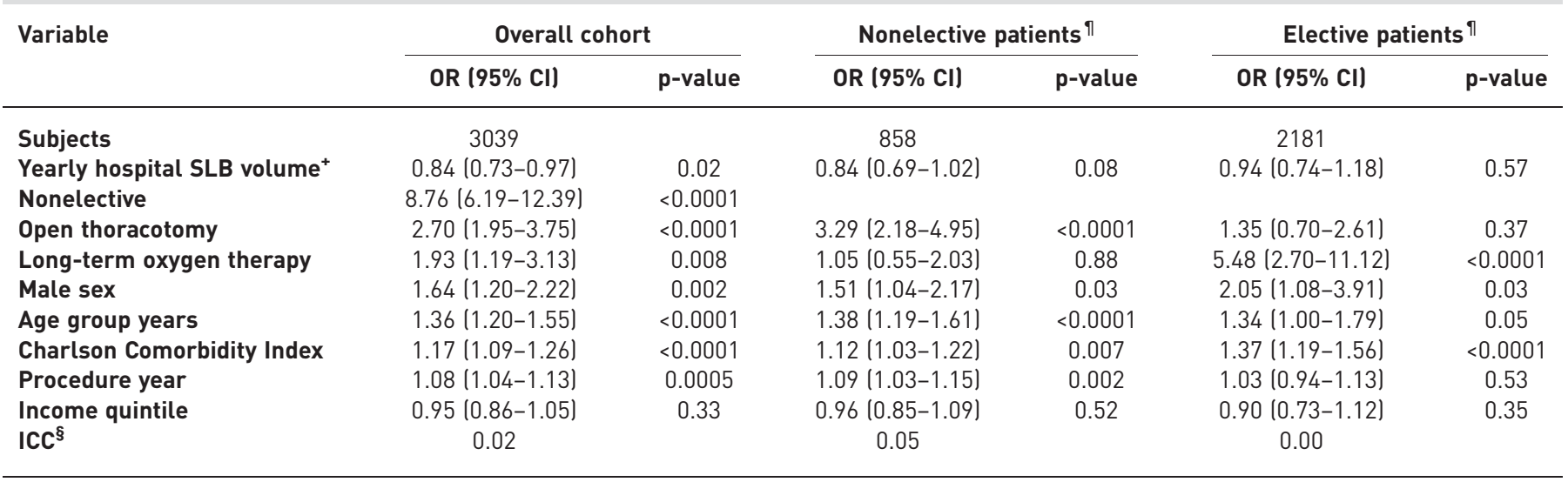

ICC: intraclass correlation coefficient. There were 18, 2 and 16 patients excluded from the overall cohort, nonelective patients and elective patients, respectively, due to missing data. $\mathrm{OR}>1$ represents higher odds of 30 -day post-operative mortality, OR $<1$ represents lower odds of 30 -day post-operative mortality. Age and income were analysed as ordinal variables, with increasing age group being associated with higher 30-day post-operative mortality in the overall cohort and nonelective patients. Age groups used were 18-29, 30-39, 40-49, 50-59, 60-69 and $\geqslant 70$ years. Charlson Comorbidity Index and procedure year were analysed as continuous variables, with higher Charlson Comorbidity Index being associated with higher 30 -day post-operative mortality in all three groups, and later procedure year being associated with higher 30 -day post-operative mortality in the overall cohort and nonelective patients. All other variables were analysed as categorical (yes versus no). ${ }^{\#}$ : the full multilevel model includes hospital (yearly hospital SLB volume) and patient-level covariates; " : an interaction term for yearly hospital SLB volume and nonelective versus elective patients was nonsignificant; ${ }^{+}$: unit for OR is per $50 \mathrm{SLBs}$ performed; ${ }^{\S}$ : ICC represents the variability in 30 -day post-operative mortality that is accounted for by hospital differences le.g. in the overall cohort, $2 \%$ of the variability in 30 -day post-operative mortality is accounted for by between-hospital differences, leaving $98 \%$ of the variability attributable to patient characteristics). 
compared with the overall cohort (OR 0.94, 95\% CI 0.74-1.18; $\mathrm{p}=0.57$, unit for OR is per 50 SLBs), although there was still a trend towards lower mortality. The ICCs were negligible, suggesting variability in 30-day post-operative mortality was accounted for by patient characteristics as opposed to between-hospital differences.

Older age group, male sex, nonelective procedure, long-term oxygen therapy, later year of procedure, higher Charlson Comorbidity Index and open thoracotomy were significantly associated with a greater odds of 30-day post-operative mortality in the overall cohort. The odds of death within 30 days were 8.76 times higher in nonelective procedures compared with elective procedures (OR 8.76, 95\% CI 6.19-12.39; $\mathrm{p}<0.0001)$. Interestingly, open thoracotomy, older age and later procedure year remained significantly associated with 30-day post-operative mortality in nonelective patients only, while long-term oxygen therapy remained significantly associated with 30-day post-operative mortality in only elective patients on stratified analysis. Results of the null and hospital-level models are shown in supplementary table S7. Adjusted odds ratios for each year of procedure in the overall cohort are shown in supplementary figure S1.

Assessment of model fit yielded excellent c-statistics of $0.86,0.86$ and 0.80 for the overall cohort, nonelective and elective patients, respectively. The Hosmer-Lemeshow goodness-of-fit test was not significant $(\mathrm{p}=0.50, \mathrm{p}=0.50$ and $\mathrm{p}=0.84$ for the overall cohort, nonelective and elective patients, respectively), suggesting the models were well calibrated.

\section{SLB volume by quartiles}

Range of yearly hospital SLB volume by increasing quartile was 1-34, 35-73, 74-143 and 144-258. The difference in 30 -day mortality was greatest between extremes of volume (10.0\% in quartile 1 versus $3.2 \%$ in quartile 4). Model results analysing yearly hospital SLB volume by quartiles for the overall cohort and stratified by nonelective and elective patients are shown in supplementary table S8. In the overall cohort, the odds of death within 30 days after SLB were significantly lower for the highest volume (fourth) quartile (OR 0.45, 95\% CI 0.25-0.79; $\mathrm{p}=0.005$, reference is quartile 1 , unit for OR is per 50 SLBs). No significant difference was seen for the second and third volume quartiles compared with quartile 1 , although there was a trend towards lower odds of 30-day post-operative mortality for the higher-volume quartiles.

\section{Discussion}

Previous research has shown that mortality after SLB is variable, and may be unacceptably high for some patients and in some settings [5-9]. We used multilevel modelling to demonstrate that higher hospital SLB volume was associated with lower 30-day post-operative mortality after SLB in a large cohort of patients with ILD. The stratified analysis suggested this relationship was stronger for nonelective patients and may be less likely to apply in elective situations. The highest volume quartile group was significantly associated with a lower odds of 30-day post-operative mortality, resulting in a volume threshold of 144 SLBs per hospital per year. It is likely that different volume thresholds could be safely applied to elective versus nonelective SLBs; however, we were not powered to assess this. To the best of our knowledge this is the first study to evaluate the volume-outcome relationship for SLB in ILD. The variability in post-operative mortality attributable to between-hospital differences was small, suggesting that the wide range of mortality described in the literature may be explained by patient-level factors. Specifically, nonelective procedure appears to be the most important prognosticator of post-operative outcome.

Similar volume-outcome relationships have been found with many surgeries and have been used to support the regionalisation of care for certain procedures such as lung cancer resection [11, 15]. The volume-outcome relationship is likely more complicated than purely surgical expertise and there are several factors specific to SLB in ILD patients to consider. Previous studies have suggested that an acute exacerbation of ILD (AE-ILD) may be precipitated by SLB and contribute to high mortality rates seen in this population $[8,17]$. In-hospital mortality from AE-ILD is as high as $50-80 \%$ [18-20]. The trigger for an AE-ILD after SLB is unclear and may be related to factors other than surgical expertise. For example, hyperoxia and mechanical stress during ventilation of the fibrotic lung and intra-operative fluid balance have been proposed as potential aetiological factors in AE-ILD after SLB [21, 22]. Institutional factors may also influence the volume-outcome relationship, with higher-volume centres having processes in place that better equip them for the peri-operative management of these patients. The experience of support staff working in the operating room, post-operation unit, intensive care unit and surgical ward may all play a role in patient outcome. Higher-volume centres may also be more likely to have ILD experts that influence patient referral and selection for SLB. Given the volume-outcome association we observed appeared to be stronger for nonelective cases, variations on the basis of expertise within hospitals are perhaps not unexpected. However, identifying the mechanisms driving a specific volume-outcome relationship remains important for implementing system changes aimed at improving patient outcomes. 
The 30 -day post-operative mortality was $7.1 \%$ in our overall study cohort. Mortality was much higher for nonelective procedures $(20.2 \%)$ compared with elective procedures (1.9\%). These results are consistent with those seen in US administrative data, where in-hospital mortality was $6.4 \%$ for a large cohort of ILD patients undergoing SLB and mortality for nonelective procedures was much higher than for elective procedures (16.0\% versus $1.7 \%$, respectively) [9]. The authors of that study were unable to ascertain 30 -day mortality, but our data on 30-day mortality would be expected to be higher than in-hospital mortality, suggesting our data are likely generalisable to other settings in North America. Interestingly, 30-day mortality after SLB for ILD in administrative data from the UK was similar to ours among elective procedures $(1.5 \%)$, but lower $(6.3 \%)$ in nonelective procedures [10]. This mortality difference may reflect variation in practice and nonelective patient selection patterns between the UK and North America. Regardless, a 30-day post-operative mortality rate of $\sim 2 \%$ for elective SLB is consistent across European and North American data.

In keeping with previous research, we also found several patient-level factors that were independently associated with 30-day post-operative mortality on multilevel modelling $[9,10]$. These included older age, male sex, higher Charlson Comorbidity Index, long-term oxygen therapy, nonelective procedure and open thoracotomy. The ICC was negligible in all three of the full multilevel models (overall cohort, nonelective and elective patients). The lack of variability in outcome related to hospital variation suggests that patient characteristics are important drivers in post-operative mortality after SLB for ILD and should be carefully considered when assessing operative risk. Nonelective procedures had a strikingly higher 30-day mortality compared with elective procedures. It is difficult to know whether this finding is attributable to high baseline risk and post-operative complications or if these patients would have died regardless because of their clinical trajectory. In either case, the clear risk of SLB in nonelective patients with ILD should be carefully weighed against the potential a SLB will significantly alter treatment decisions and clinical course before deciding to proceed in such individuals.

Interestingly, while unadjusted mortality appeared to be stable over time, we found that later procedure year was associated with higher odds of SLB mortality on multilevel modelling in the overall cohort and nonelective patients. At first glance this is counterintuitive as operative mortality has fallen over time for many procedures, such as aortic valve replacement, carotid endarterectomy and several cancer resections $[23,24]$. One hypothesis for the unexpected finding is that clinicians have become more aggressive with pursuing SLB over time. Our patient population appeared similar over time regarding age, sex and comorbidity burden. We were limited in our ability to determine the severity of lung disease, but the use of long-term oxygen therapy increased over time, suggesting that patients may have had progressively more severe lung disease during the study period. Several key publications on ILD occurred during our study period that may have influenced trends in pursuing SLB. In 2002, the first international guideline on the diagnosis and classification of ILDs was published jointly by the American Thoracic Society and European Respiratory Society. This guideline placed an emphasis on obtaining a SLB to aid in diagnosis in the absence of contraindications and noted that with the advent of VATS, SLB should be associated with less risk than historically seen [1]. Furthermore, this guideline stated that transbronchial biopsies were generally not useful and discouraged the traditional practice of a trial of therapy to determine prognosis rather than pursuing SLB. The authors also made note of the low SLB rate in patients with ILD at that time. In 2011, interim results from the PANTHER trial were available showing increased mortality in idiopathic pulmonary fibrosis (IPF) patients treated with prednisone, azathioprine and $N$-acetylcysteine $[25,26]$, providing further discouragement for the clinical practice of a trial of immunosuppressive therapy in situations of diagnostic uncertainty. In 2012, pirfenidone, an antifibrotic agent, was approved for use in IPF by Health Canada and, since this class of medication is not indicated in non-IPF ILD, further emphasis may have been placed on the importance of SLB after 2012 [27]. It is possible that these changes to the landscape of ILD in Canada resulted in clinicians pursuing SLB more aggressively in higher-risk patients over time and thus increasing post-operative mortality. Specifically, it is possible that patients with IPF were biopsied more frequently in recent years and were more likely to have higher post-operative mortality [9]. Expanding on the recent IPF diagnostic guidelines [28] with the development of a statement advising physicians on when SLB is contraindicated and outlining specific pre-operative considerations should be considered to avoid potentially harmful trends associated with inappropriate SLBs.

There are several limitations to our study. First, large-scale validation studies in ICES databases have not been performed for ILD codes. As a result, we were unable to assess the relationship between ILD subtype and 30-day post-operative mortality after SLB. However, we felt the specificity of an ILD diagnosis in our cohort was likely to be high, given that individuals had both a diagnostic code for ILD and underwent a SLB. Second, there is always potential for unmeasured confounding when using administrative databases for research. We were unable to ascertain lung function or intra-operative ventilator management. These may be important factors in outcome after SLB in ILD and difficult to tease out given the limitations of 
administrative data. Third, the stratified analysis should be interpreted with caution given an interaction term for surgical volume and nonelective versus elective procedure was nonsignificant and the sample size was not large enough to excluded a volume-outcome relationship for elective procedures. Lastly, while we showed a volume-outcome relationship between SLB and post-operative mortality, recommending system changes aimed at improving outcomes remains a challenge. We cannot be sure of the exact mechanisms behind the volume-outcome relationship. Surgical expertise is an attractive and straightforward theory which can lead to minimum volume recommendations for procedures. However, previous studies have found that increasing surgical volume does not always translate into a decrease in post-operative mortality [15], suggesting the relationship is more complicated than the research can sometimes elucidate. Despite these limitations, we were able to reliably ascertain 30-day post-operative mortality, a clinically meaningful end-point, expanding on previous administrative data that have relied on in-hospital mortality estimates.

We applied multilevel modelling to show that higher yearly hospital SLB volume is associated with lower 30-day mortality after SLB for ILD. Nonelective SLB was associated with a significantly higher mortality rate than elective SLB and should therefore be discouraged unless there is a clear indication.

Acknowledgements: This study made use of de-identified data from the ICES Data Repository, which is managed by the Institute for Clinical Evaluative Sciences with support from its funders and partners: Canada's Strategy for Patient-Oriented Research (SPOR), the Ontario SPOR Support Unit, the Canadian Institutes of Health Research and the Government of Ontario. The opinions, results and conclusions reported are those of the authors. No endorsement by ICES or any of its funders or partners is intended or should be inferred. Parts of this material are based on data and information compiled and provided by the Canadian Institute for Health Information (CIHI). However, the analyses, conclusions, opinions and statements expressed herein are those of the author, and not necessarily those of CIHI.

Conflict of interest: J.H. Fisher reports a patient donation to the University Heath Network Foundation Interstitial Lung Disease Research Fund, during the conduct of the study. S. Shapera reports a patient donation to the University Heath Network Foundation Interstitial Lung Disease Research Fund, during the conduct of the study; personal fees from AstraZeneca and Amgen, and grants and personal fees from Boehringer Ingelheim and Hoffman-La Roche, outside the submitted work. T. To reports grants from Ontario Ministry of Health and Long-term Care, Ontario Ministry of the Environment and Climate Change, CIHR (Canadian Institute for Health Research), Health Canada and CRRN (Canadian Respiratory Research Network), outside the submitted work. T.K. Marras reports a patient donation to the University Heath Network Foundation Interstitial Lung Disease Research Fund, during the conduct of the study; grants and personal fees from Insmed, and personal fees from AstraZeneca, Horizon and RedHill, outside the submitted work. A. Gershon has nothing to disclose. S. Dell has nothing to disclose.

Support statement: Financial support for this study was provided by a patient donation to the University Health Network Foundation. Funding information for this article has been deposited with the Crossref Funder Registry.

\section{References}

1 American Thoracic Society, European Respiratory Society. American Thoracic Society/European Respiratory Society international multidisciplinary consensus classification of the idiopathic interstitial pneumonias. Am J Respir Crit Care Med 2002; 165: 277-304.

2 Travis WD, Costabel U, Hansell DM, et al. An official American Thoracic Society/European Respiratory Society statement: update of the international multidisciplinary classification of the idiopathic interstitial pneumonias. Am J Respir Crit Care Med 2013; 188: 733-748.

3 Raghu G, Collard HR, Egan JJ, et al. An official ATS/ERS/JRS/ALAT statement: idiopathic pulmonary fibrosis: evidence-based guidelines for diagnosis and management. Am J Respir Crit Care Med 2011; 183: 788-824.

4 Bradley B, Branley HM, Egan JJ, et al. Interstitial lung disease guideline: the British Thoracic Society in collaboration with the Thoracic Society of Australia and New Zealand and the Irish Thoracic Society. Thorax 2008; 63: Suppl. 5, v1-v58.

5 Carrillo G, Estrada A, Pedroza J, et al. Preoperative risk factors associated with mortality in lung biopsy patients with interstitial lung disease. J Invest Surg 2005; 18: 39-45.

6 Lettieri CJ, Veerappan GR, Helman DL, et al. Outcomes and safety of surgical lung biopsy for interstitial lung disease. Chest 2005; 127: 1600-1605.

7 Utz JP, Ryu JH, Douglas WW, et al. High short-term mortality following lung biopsy for usual interstitia pneumonia. Eur Respir J 2001; 17: 175-179.

8 Kreider ME, Hansen-Flaschen J, Ahmad NN, et al. Complications of video-assisted thoracoscopic lung biopsy in patients with interstitial lung disease. Ann Thorac Surg 2007; 83: 1140-1144

9 Hutchinson JP, Fogarty AW, McKeever TM, et al. In-hospital mortality after surgical lung biopsy for interstitial lung disease in the United States. 2000 to 2011. Am J Respir Crit Care Med 2016; 193: 1161-1167.

10 Hutchinson JP, McKeever TM, Fogarty AW, et al. Surgical lung biopsy for the diagnosis of interstitial lung disease in England: 1997-2008. Eur Respir J 2016; 48: 1453-1461.

11 Birkmeyer JD, Siewers AE, Finlayson EV, et al. Hospital volume and surgical mortality in the United States. N Engl J Med 2002; 346: 1128-1137.

12 Urbach DR, Stukel TA, Croxford R, et al. Analysis of Current Research Related to the Impact of Low-volume Procedures/Surgery and Care on Outcomes of Care. Toronto, Canadian Institute for Health Information, 2004.

13 Halm EA, Lee C, Chassin MR. Is volume related to outcome in health care? A systematic review and methodologic critique of the literature. Ann Intern Med 2002; 137: 511-520.

14 Dudley RA, Johansen KL, Brand R, et al. Selective referral to high-volume hospitals: estimating potentially avoidable deaths. JAMA 2000; 283: 1159-1166. 
15 Finley CJ, Bendzsak A, Tomlinson G, et al. The effect of regionalization on outcome in pulmonary lobectomy: a Canadian national study. J Thorac Cardiovasc Surg 2010; 140: 757-763.

16 Charlson ME, Pompei P, Ales KL, et al. A new method of classifying prognostic comorbidity in longitudinal studies: development and validation. J Chronic Dis 1987; 40: 373-383.

17 Rotolo N, Imperatori A, Dominioni L, et al. Efficacy and safety of surgical lung biopsy for interstitial disease. Experience of 161 consecutive patients from a single institution in Italy. Sarcoidosis Vasc Diffuse Lung Dis 2015; 32: $251-258$.

18 Kim DS, Park JH, Park BK, et al. Acute exacerbation of idiopathic pulmonary fibrosis: frequency and clinical features. Eur Respir J 2006; 27: 143-150.

19 Song JW, Hong SB, Lim CM, et al. Acute exacerbation of idiopathic pulmonary fibrosis: incidence, risk factors and outcome. Eur Respir J 2011; 37: 356-363.

20 Al-Hameed FM, Sharma S. Outcome of patients admitted to the intensive care unit for acute exacerbation of idiopathic pulmonary fibrosis. Can Respir J 2004; 11: 117-122.

21 Ghatol A, Ruhl AP, Danoff SK. Exacerbations in idiopathic pulmonary fibrosis triggered by pulmonary and nonpulmonary surgery: a case series and comprehensive review of the literature. Lung 2012; 190: 373-380.

22 Mizuno $\mathrm{Y}$, Iwata $\mathrm{H}$, Shirahashi $\mathrm{K}$, et al. The importance of intraoperative fluid balance for the prevention of postoperative acute exacerbation of idiopathic pulmonary fibrosis after pulmonary resection for primary lung cancer. Eur J Cardiothorac Surg 2012; 41: e161-e165.

23 Barreto-Filho JA, Wang Y, Dodson JA, et al. Trends in aortic valve replacement for elderly patients in the United States, 1999-2011. JAMA 2013; 310: 2078-2085.

24 Finks JF, Osborne NH, Birkmeyer JD. Trends in hospital volume and operative mortality for high-risk surgery. N Engl J Med 2011; 364: 2128-2137.

25 Idiopathic Pulmonary Fibrosis Clinical Research Network. Prednisone, azathioprine, and $\mathrm{N}$-acetylcysteine for pulmonary fibrosis. N Engl J Med 2012; 366: 1968-1977.

26 National Institutes of Health. Commonly used three-drug regimen for idiopathic pulmonary fibrosis found harmful. 2011. www.nih.gov/news/health/oct2011/nhlbi-21.htm Date last accessed: June 20, 2018.

27 Health Canada. Summary basis of decision for Esbriet. 2016. https://hpr-rps.hres.ca/reg-content/summary-basis-decisiondetailTwo.php?linkID=SBD00213 Date last accessed: June 20, 2018.

28 Raghu G, Remy-Jardin M, Myers JL, et al. Diagnosis of idiopathic pulmonary fibrosis. An Official ATS/ERS/JRS/ ALAT Clinical Practice Guideline. Am J Respir Crit Care Med 2018; 198: e44-e68. 